\title{
Synthesis of variable-interval performance from those for component fixed-interval schedules of reinforcement*
}

\author{
DAVID A. ECKERMAN \\ University of North Carolina, Chapel Hill, North Carolina 27514 \\ and \\ ROBERT D. HIENZ $\dagger$ \\ University of Washington Medical School, Seattle, Washington 19805
}

\begin{abstract}
Two pigeons were trained to peck a response key under a procedure in which 5-min periods of keylight-on alternated with 6-min periods of blackout. During keylight-on periods, food reinforcement was provided for the first peck following $20 \mathrm{sec}$ (E schedule), $120 \mathrm{sec}$ (M schedule), or $300 \mathrm{sec}$ (L schedule) into the period. In different experimental conditions, the $\mathrm{E}, \mathrm{M}$, and $\mathrm{L}$ schedules were presented either alone or in combination. When $E$ and/or $M$ schedules were presented, food reinforcement was provided in only a portion (usually one-half) of the periods. Performance under the combination conditions was roughly predicted by summing the separate E-, M-, and L-schedule performances. Deviations from adequate prediction were, however, observed, which demonstrated interactions between the $\mathrm{E}, \mathrm{M}$, and $\mathrm{L}$ schedules.
\end{abstract}

Catania and Reynolds (1968) presented evidence that the rate of responding at time y following reinforcement in a variable-interval (VI) schedule of reinforcement was controlled by the local probability of reinforcement at time $y$. The local probability of reinforcement was defined as the number of times reinforcement was obtained within the temporal interval $t_{x}-t_{z}$ (including time y) following the previous reinforcement and dividing by the total number of times the interval $t_{x}-t_{z}$ was traversed. When the number of possible intervals comprising the VI schedule was small, however, this calculating formula became inapplicable, for it did not acknowledge the "extensive period of time since reinforcement over which a particular probability of reinforcement can have its effect and the degree to which a later high probability of reinforcement can influence the local rate of responding maintained by an early low probability of reinforcement [Catania \& Reynolds, 1968, p. 364]."

A related formulation appeared, roughly, to apply to VI schedules comprising just a few component intervals. "To some extent, the performances maintained by the present schedules can be considered simple combinations of the performances separately maintained by the component FI (fixed interval) schedules [Catania \& Reynolds, 1968, p. 364]."

The present experiment further examines the degree to which VI performance may be "synthesized" from performances under the component FI schedules. The

*The work was supported by Grant VF-311 from the University Research Council of the University of North Carolina. The authors are indebted to $S$. Stern for his aid in performing and analyzing data from the experiment. Reprints are available from the first author, Department of Psychology, University of North Carolina, Chapel Hill, North Carolina 27514.

+Present address: Department of Biomedical Engineering, The Johns Hopkins School of Medicine, Baltimore, Maryland 21205. present procedure was, however, somewhat different from that used by Catania and Reynolds. Each component interval was separately initiated by reinforcement in their study (mix FIFI). In the present work, component intervals were initiated following an extended $(6 \mathrm{~min})$ blackout, and all component FI schedules were simultaneously initiated (conc. FIFI). Reinforcement occurred probabilistically at the end of each of the concurrently programmed component intervals. From this procedure, it is thus possible to determine the effect of reinforcement availability at one point in the keylight-on period on rate of responding throughout the period.

\section{METHOD}

\section{Subjects}

Two male White Carneaux pigeons, maintained at $75 \%$ of free-feeding weight, served as Ss. When not in the experimental chamber, they were housed in individual home cages with water and grit available. Diet consisted of Purina Wild Bird Seed, with sunflower seeds removed (reinforcement mixture), supplemented as needed by Purina Pigeon Chow.

\section{Apparatus}

A standard one-key operant chamber for pigeons was used. The key was back illuminated by white light, which was turned off briefly by each peck. No hou selight was used, and no water was available in the chamber. Activation of the grain feeder for $5 \mathrm{sec}$ served as reinforcement. Reinforcement schedules were programmed using stepping switches and clocks.

\section{Procedure}

Prior History. Both birds had extended (approximately 500 sessions) exposure to a FI 5-min schedule of reinforcement for keypecking (Eckerman \& McGourty, 1969). During this training, each FI was preceded by a $30-\mathrm{sec}$ blackout of the chamber, and sessions consisted of 100 FI 5-min intervals.

Present Experiment. Sessions in the present experiment 
Table 1

Experimental Conditions

\begin{tabular}{lccccc}
\hline & & & & \multicolumn{2}{c}{ Number of Sessions } \\
\cline { 5 - 6 } Condition & $P(E)$ & $P(M)$ & $P(L)$ & Bird 52 & Bird 53 \\
\hline 1-L & 0.00 & 0.00 & 1.00 & 33 & 44 \\
2-E+L & 0.22 & 0.00 & 1.00 & 37 & 27 \\
3-E & 1.00 & 0.00 & 0.00 & 22 & 22 \\
4-E & 0.75 & 0.00 & 0.00 & 8 & 9 \\
5-E & 0.50 & 0.00 & 0.00 & 4 & 0 \\
6-L & 0.00 & 0.00 & 1.00 & 49 & 46 \\
7-E+M+L & 0.50 & 0.50 & 1.00 & 47 & 46 \\
8-M+L & 0.00 & 0.50 & 1.00 & 22 & 22 \\
9-E+M & 0.50 & 0.50 & 0.00 & 26 & 26 \\
10-M & 0.00 & 0.50 & 0.00 & 21 & 20 \\
11-E+L & 0.50 & 0.00 & 1.00 & 18 & 18 \\
12-L & 0.00 & 0.00 & 1.00 & 65 & 64 \\
13-E+M+L & 0.50 & 0.50 & 1.00 & 34 & 35 \\
\hline
\end{tabular}

consisted of 50 alternations of a cycle of keylight-on for 5 min followed by keylight-off for $6 \mathrm{~min}$. Reinforcement was available only during keylight-on periods, with the first peck following either $20 \mathrm{sec}$ (E schedule), $120 \mathrm{sec}$ (M schedule), or $300 \mathrm{sec}$ (L schedule) into the keylight-on period, producing the reinforcer with a probability $\mathrm{p}$. These three schedules were presented both singly and concurrently with each other, producing seven experimental conditions: E, M, L, EM, EL, and EML. When an $E$ and/or $M$ schedule was employed, the probability of reinforcement under these two schedules was normally 0.50 , with independent scrambled sequences determining which of the 50 intervals would contain reinforcement availabilities for each of the two schedules. Also, a limited hold of $60 \mathrm{sec}$ was imposed on the reinforcement availabilities set up by the $E$ and $M$ schedules. For sessions in which the $\mathrm{L}$ schedule operated, the first peck following $5 \mathrm{~min}$ of keylight-on always produced reinforcement and initiated the blackout. If no L schedule was programmed, the blackout period started automatically $5 \mathrm{~min}$ after keylight-on, provided at least one peck had occurred in the interval, else blackout commenced with the next peck. Table 1 lists the exact order of experimental conditions, including the probability of reinforcement availability for each of the three basic schedules of a condition.

Data were collected as follows: The number of pecks occurring during sequential 20 -sec time bins within the 5-min keylight-on periods was totaled across each session for those intervals which did not contain $E$ or $M$ reinforcements. Rates of responding measured for each portion of the 5-min intervals were thus uncontaminated by interruptions in on-going behavior due to reinforcement delivery.

The experimental plan consisted of examining the degree to which response levels maintained by each of the three basic schedules presented singly would sum to reproduce the response levels obtained when the schedules were run concurrently. For example, the EML schedule (all three schedules operating concurrently) resembles a variable-interval schedule of reinforcement in that reinforcement might occur at $20 \mathrm{sec}$, might occur at $120 \mathrm{sec}$, and always occurs at $300 \mathrm{sec}$ into the interval (i.e., just as there is a maximum interreinforcement interval in a variable-interval schedule, so is there one here). The degree to which this EML schedule performance might then be "synthesized" from the single schedule performances was then assessed by summing the corresponding local response rates generated under the singly run $\mathrm{E}, \mathrm{M}$, and $\mathrm{L}$ schedules, and comparing this synthesized performance with the actual performance.

\section{RESULTS AND DISCUSSION}

Data are provided in Fig. 1 to show performances generated by $\mathrm{E}$ (Condition 4), M (Condition 10) and L (average of Conditions 6 and 12) reinforcement schedules. The function for the $L$ schedule shows a well-maintained FI performance. Less behavior was maintained by the $E$ and $M$ schedules, where maximum rates of responding ranged only from 0.20 to 0.42 responses per second. The performance for the $M$ schedule represents a well-maintained low rate of pecking. Performance was not adequately maintained, however, for the $\mathrm{E}$ schedule even in Condition 4 in which three-quarters of the light-on intervals contained a reinforcement. Data were taken from Sessions 3-5 of Condition 4, where pecking was still present. Data for all other conditions to be presented were averaged from the last five sessions of the condition.

For $\mathrm{E}, \mathrm{M}$, and $\mathrm{L}$ schedules, rate of responding was maximal at or just after the time in the light-on interval when reinforcement was due. Yet, responding was maintained for each of these schedules throughout the light-on interval. Reinforcement at any temporal position in the interval thus maintained some responding throughout.

Data from multiple reinforcement conditions (EML, EM, and ML) are presented in the left column of Fig. 2 . Again, there is evidence both for an especially strong rate increasing effect of a reinforcement surrounding its temporal position in the interval as well as a continued rate increasing effect of that reinforcement throughout the interval (for example, rate of responding is substantial at the end of intervals for the EM schedule even though $\mathrm{L}$ reinforcements never occurred).

The proposal that the performance generated by the multiple-reinforcement schedules can be synthesized from the performance generated by the component single-reinforcement schedules may be evaluated from

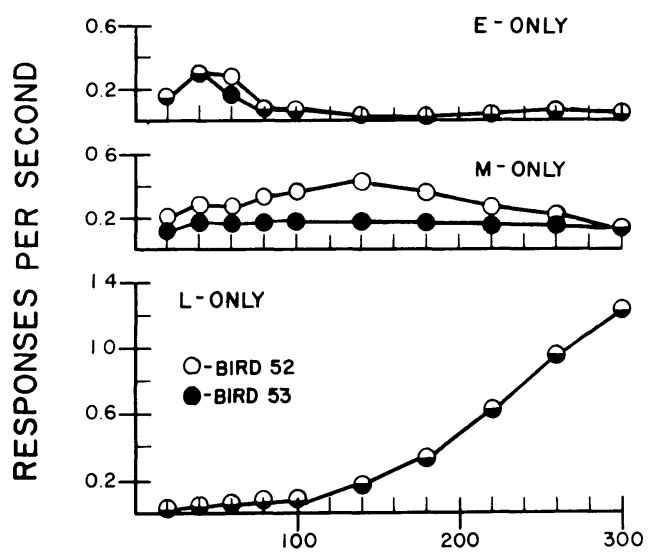

TIME IN INTERVAL (SEC)

Fig. 1. Rate of responding during successive periods in the keylight-on intervals for the conditions indicated. Half-filled circles indicate points where the value for the two birds differed by less than 0.05 responses per second. 
Fig. 2. Rate of responding during successive periods in the keylight-on intervals for the conditions noted (left column); sums of rates of responding obtained during these periods in the indicated single-schedule conditions (middle column); and difference between left and middle column values (right column). Half-filled circles indicate points where the value for the two birds differed by less than 0.05 responses per second.
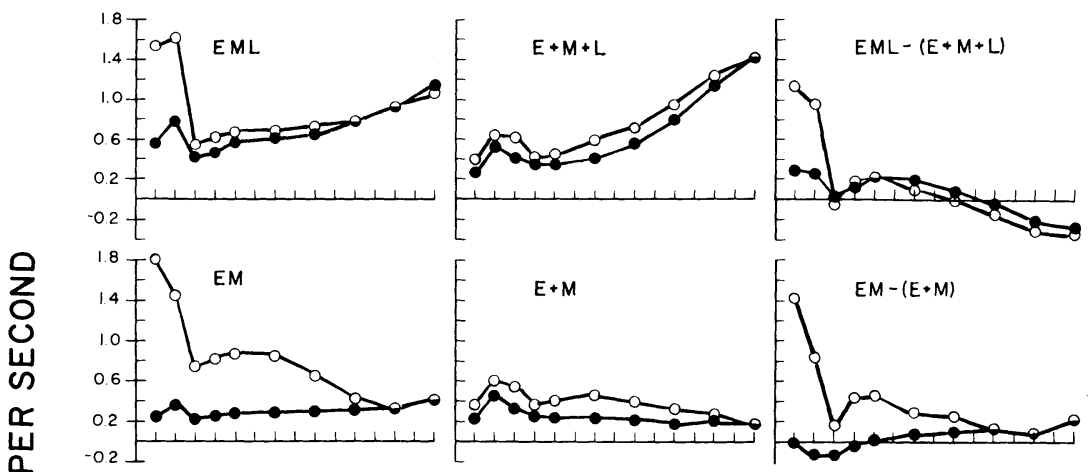

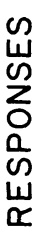
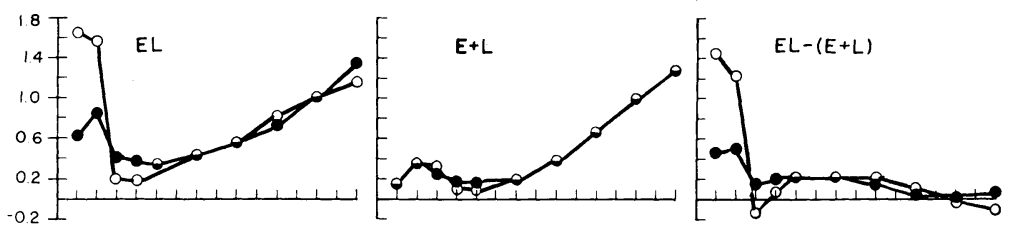

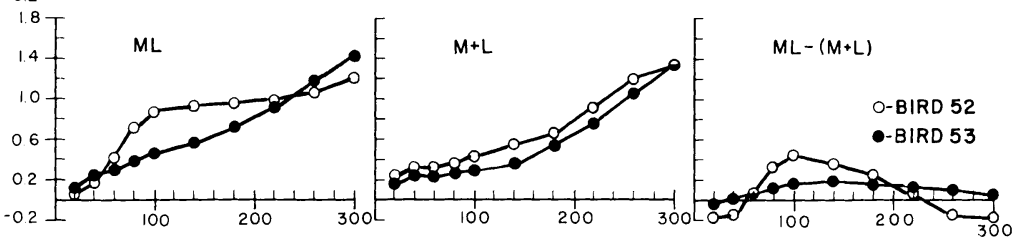

TIME IN INTERVAL (SEC)

the middle and right columns of Fig. 2 . In the middle column, rates of pecking from single-reinforcement schedule performances have been summed. In the right column, the difference between the actual and synthesized (summed) performances is shown.

The following comments derive from inspection of Fig. 2. First, the general forms of the functions are roughly predicted by the synthesis (especially true for Bird 53). Second, $\mathrm{E}$ and $\mathrm{M}$ reinforcements are more effective in increasing rate of responding when presented concurrently with other schedules than predicted by the synthesis (especially true for Bird 52). That is, the general error of prediction is that the "synthesized" rate is too low. The only exception to this general trend is the very close synthesis predictions for the EM condition for Bird 53. Third, the $\mathrm{L}$ reinforcements were about equally effective in increasing rate of responding for multiple-reinforcement conditions and syntheses, except when both $\mathrm{E}$ and $\mathrm{M}$ reinforcements were provided. In the EML condition, that is, responding was lower near the end of the interval than predicted by the synthesis.

It would seem, then, that the proposal that a variable-interval performance is a sum of its component fixed-interval performances has general merit, since the form of the multiple-reinforcement schedule functions were roughly predicted by the synthesis from component schedules. What is left out of this proposal is an interaction between these component schedules in which, apparently, reinforcements provided after short intervals are more effective when they exist in a context of long intervals than when they exist alone. There is also a hint in the present data that reinforcements provided after long intervals may be made less effective by a context of shorter intervals.

Lastly, the fact that reinforcement at any point in the light-on intervals in the present experiment affected performance throughout the interval argues against the proposal tentatively forwarded and partially retracted by Catania and Reynolds (1968), that reinforcement affects responding only over a portion of the range of possible interreinforcement intervals. Rather, the probability of reinforcement at Time $X$ seems to affect responding at all times throughout the range interreinforcement intervals.

\section{REFERENCES}

Catania, A. C., \& Reynolds, G. S. A quantitative analysis of responding maintained by interval schedules of reinforcement. Journal of the Experimental Analysis of Behavior, 1968, 11, 327-383.

Eckerman, D. A., \& McGourty, D. P. Varying temporal placement of response-produced stimuli in a fixed-schedule. Psychonomic Science, 1969, 15, 225-227.

(Received for publication November 14, 1973.) 